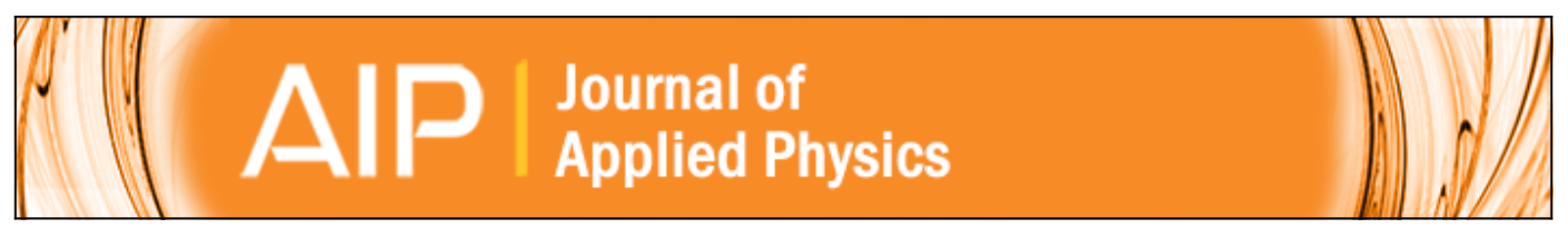

Verification of band offsets and electron effective masses in GaAsN/GaAs quantum wells: Spectroscopic experiment versus 10 -band $k \cdot p$ modeling

K. Ryczko, G. Sk, P. Sitarek, A. Mika, J. Misiewicz, F. Langer, S. Höfling, A. Forchel, and M. Kamp

Citation: Journal of Applied Physics 113, 233508 (2013); doi: 10.1063/1.4810920

View online: http://dx.doi.org/10.1063/1.4810920

View Table of Contents: http://scitation.aip.org/content/aip/journal/jap/113/23?ver=pdfcov

Published by the AIP Publishing

AlP Re-register for Table of Content Alerts 


\title{
Verification of band offsets and electron effective masses in GaAsN/GaAs quantum wells: Spectroscopic experiment versus 10-band $k \cdot p$ modeling
}

\author{
K. Ryczko, ${ }^{1}$ G. Sęk, ${ }^{1}$ P. Sitarek, ${ }^{1}$ A. Mika, ${ }^{1}$ J. Misiewicz, ${ }^{1}$ F. Langer, ${ }^{2}$ S. Höfling, ${ }^{2}$ \\ A. Forchel, ${ }^{2}$ and M. Kamp ${ }^{2}$ \\ ${ }^{1}$ Institute of Physics, Wroclaw University of Technology, Wybrzeże Wyspiańskiego 27, 50-370 Wroclaw, \\ Poland \\ ${ }^{2}$ Technische Physik, Physikalisches Institut and Wilhelm-Conrad-Röntgen-Research Center for Complex \\ Material Systems, University of Würzburg, Am Hubland, D-97074 Würzburg, Germany
}

(Received 1 March 2013; accepted 29 May 2013; published online 19 June 2013)

\begin{abstract}
Optical transitions in $\mathrm{GaAs}_{1-\mathrm{x}} \mathrm{N}_{\mathrm{x}} / \mathrm{GaAs}$ quantum wells (QWs) have been probed by two complementary techniques, modulation spectroscopy in a form of photoreflectance and surface photovoltage spectroscopy. Transition energies in QWs of various widths and $\mathrm{N}$ contents have been compared with the results of band structure calculations based on the 10-band $\mathbf{k} \cdot \mathbf{p}$ Hamiltonian. Due to the observation of higher order transitions in the measured spectra, the band gap discontinuities at the GaAsN/GaAs interface and the electron effective masses could be determined, both treated as semi-free parameters to get the best matching between the theoretical and experimental energies. We have obtained the chemical conduction band offset values of $86 \%$ for $\mathrm{x}=1.2 \%$ and $83 \%$ for $\mathrm{x}=2.2 \%$, respectively. For these determined band offsets, the electron effective masses equal to about $0.09 \mathrm{~m}_{\mathrm{o}}$ in QWs with $1.2 \% \mathrm{~N}$ and $0.15 \mathrm{~m}_{\mathrm{o}}$ for the case of larger $\mathrm{N}$ content of 2.2\%. (C) 2013 AIP Publishing LLC. [http://dx.doi.org/10.1063/1.4810920]
\end{abstract}

\section{INTRODUCTION}

Dilute nitride alloys such as GaAsN have attracted a great deal of experimental and theoretical attention in the last decade due to their unusual physical properties. One example is a drastically reduced bandgap with the incorporation of a small amount of nitrogen into GaAs. ${ }^{1,2}$ This fact made these materials technologically attractive for optoelectronic devices, ${ }^{3-5}$ e.g., GaAsN was used in structures designed for fast saturable absorber devices. ${ }^{6}$ Moreover, GaAsN is very often used in more complex step-like quantum well (QW) structures as InGaAsN/GaAsN/GaAs, when it serves as a strain reducing layer and helps to reach longer emission wavelengths at lower In and N contents, which is beneficial for the structural and optical material quality, and hence also for the performances of the related devices. ${ }^{7,8} \mathrm{In}$ that context, such GaAsN-containing QWs can also be imagined to be exploited in more sophisticated and futuristic structures as QWs in optical resonators, where the physical effects resulting from a manipulation of both electronic and photonic wave functions can be used to tailor the lightmatter interaction in the sense of coupling between the QW excitons and the photonic modes of the optical cavity. The latter, when combined with the use of dilute nitrides, could bring to the practical exploitation of the well-known polaritonic physics and Bose-Einstein condensation in optoelectronic devices at the telecommunication wavelengths. As we have previously shown theoretically, ${ }^{9}$ the oscillator strength of an InGsAsN/GaAsN/GaAs well, which can be used for the formation of exciton-polaritons at for instance $1.3 \mu \mathrm{m}$, is strongly and non-monotonically dependent on the value of the band gap discontinuity (and hence the related band offsets ratio) at the GaAsN/GaAs interface. Therefore, experimental determination or verification of the real band offsets for this combination of materials is a subject of the current paper. We recognize that the existing literature data concerning that aspect seems to be inconsistent or controversial. Theoretical studies based on the dielectric model predicted a type-II alignment for the GaNAs/GaAs system, ${ }^{10}$ which has further been supported by X-ray photoemission spectroscopy, ${ }^{11}$ while first-principles calculations suggest a type-I lineup in this system, ${ }^{12}$ with several following papers claiming that experimentally. ${ }^{13-17}$ Furthermore, adding nitrogen atoms into the GaAs matrix results in an enhancement of the electron effective mass, which makes the entire physical situation more complex and the proper interpretation of the optical spectra even more difficult. The issue of the effective mass has already been considered both theoretically and experimentally; ${ }^{16,18-22}$ however, currently the experimental results on the effective electron mass, especially in the case when the nitrogen $(\mathrm{N})$ mole fraction approaches or exceeds $1.5 \%$ in the GaAsN layers,${ }^{20}$ are still significantly different than predicted from the band-anticrossing (BAC) modeling ${ }^{18}$ and certain corrections need to be considered.

In this report, we studied experimentally the energies of the interband transitions in GaAsN/GaAs QWs of various widths and compositions, and then identified them theoretically exploiting the 10-band $\mathbf{k} \cdot \mathbf{p}$ model, which allowed estimating both the band offset and the electron effective masses for $\mathrm{N}$ contents useful in photonic $\mathrm{QW}$ structures emitting in the telecommunication range of $1.3 \mu \mathrm{m}$.

\section{EXPERIMENTAL}

For these studies, we used samples of $\mathrm{GaAs}_{1-\mathrm{x}} \mathrm{N}_{\mathrm{x}} / \mathrm{GaAs}$ single QWs grown by molecular beam epitaxy on a (001) GaAs substrate and a GaAs buffer, and capped with $100 \mathrm{~nm}$ of GaAs. There have been investigated two sets of structures 
with two different $\mathrm{N}$ contents (1.2\% and $2.2 \%$ of nitrogen) and various QW widths of 6,8 , and $10 \mathrm{~nm}$. The compositions of the layers are determined based on growth calibration samples. At first the $\mathrm{N}$ content was calibrated by bulk-like $\mathrm{GaAsN}$ material. The relative strain of the given composition with respect to the GaAs substrate was estimated by high resolution X-ray diffraction (HR XRD), giving a direct measure of the absolute $\mathrm{N}$ content. By using the same parameters again for the growth of the QW samples their composition could be given quite exactly. The estimated error bar is about $0.1 \%$ of the $\mathrm{N}$ content. The thicknesses of the QWs were calibrated in the same manner by accurately estimating the gallium growth rate by HR-XRD and double checking it with scanning electron microscopy measurements.

For the determination of the optical transitions, especially the higher order ones which are more sensitive to the material parameter details like the band gap discontinuities, we used two different absorption like techniques, photoreflectance-PR (a kind of modulation spectroscopy) and surface photovoltage spectroscopy-SPS, both known as efficient in detecting the entire spectrum of transitions in various, even complex and multilayered, quantum structures. ${ }^{23-27}$ In photoreflectance (or modulated reflectivity) the normalized changes of the reflectivity coefficient are measured under external modulation stemming from varying illumination with a secondary beam (usually from a laser). This gives the derivative-like spectra with enhanced sensitivity to the optical transitions occurring in the investigated structure, of even a very small oscillator strength (as the nominally parity forbidden transitions, or those in quantum dots). ${ }^{28-30}$ Photoreflectance was measured by using a standard setup in the so called bright configuration $^{31}$ when the tungsten halogen lamp and the $660 \mathrm{~nm}$ line of a semiconductor laser served as sources of the probe and pump beams, respectively. The photomodulated changes in the intensity of the reflected beam were detected by an InGaAs thermoelectrically cooled photodiode combined with a $0.5 \mathrm{~m}$ focal length monochromator dispersing the reflected light. The lock-in technique was used for the AC/DC signal processing and analog/digital conversion in order to record the spectral dependence of the normalized changes of the reflectivity coefficient $\Delta \mathrm{R} / \mathrm{R}$.

In surface photovoltage spectroscopy, the change in the surface potential due to the optically excited electron-hole pair generation under periodic illumination and subsequent carrier redistribution and/or capture in the surface states are measured. For confined state structures as QWs, it basically reflects spectrally the absorption coefficient. ${ }^{23}$ Surface photovoltage spectroscopy measurements were performed on an independent setup in a normal incidence beam configuration. The signal was measured between the sample and a reference metal grid electrode in a capacitive manner as a function of the photon energy of the probe beam. A soft contact mode was used to enhance the photovoltage signals. ${ }^{32}$ The illumination system consisted of a $200 \mathrm{~W}$ quartz-halogen lamp chopped at $180 \mathrm{~Hz}$ and a $0.3 \mathrm{~m}$ focal length grating monochromator. The photovoltage spectrum on the metal grid was measured with a copper plate as the ground electrode using a buffer circuit.

\section{THEORETICAL APPROACH}

To study the band structure of III-V semiconductor such as GaAsN we employed the $\mathbf{k} \cdot \mathbf{p}$ method. ${ }^{33}$ The strong interaction between the $\mathrm{N}$ resonant states and the conduction band states means that the conventional 8-band $\mathbf{k} \cdot \mathbf{p}$ model is not able to describe the band structure properly, especially the conduction band states of GaAsN/GaAs quantum wells. On the other hand, it has also been demonstrated that exploiting a simplified approach taking into account just the coupling between the conduction band states and the nitrogen states (so called band anticrossing model) is not satisfactory as well. It neglects the conduction and valence band interaction and does not give an accurate prediction of electron and hole higher energy confined states ${ }^{34}$ whereas the latter are used by us for comparison with the experimental results. Therefore, this leads to the necessity of using the 10-band $\mathbf{k} \cdot \mathbf{p}$ Hamiltonian, which we used after Ref. 33. We solved the Schrödinger equation using the finite difference technique. ${ }^{35}$ The material parameters used in our calculations, except for band offsets and electron effective masses which are to be determined here, are taken after Ref. 16. Further details of the used theoretical approach are described in the appendix.

\section{RESULTS AND DISCUSSION}

\section{A. Optical spectra}

In order to verify the band structure details as, e.g., the band offsets, we have used two complementary experimental techniques, both giving absorption-like spectra. These spectra allow for a highly sensitive detection of optical transitions in low-dimensional structures. ${ }^{23-27,36}$ Figure 1 shows an example of such room temperature spectra for three GaAsN/ GaAs QW samples with the nominal $\mathrm{N}$ content of about

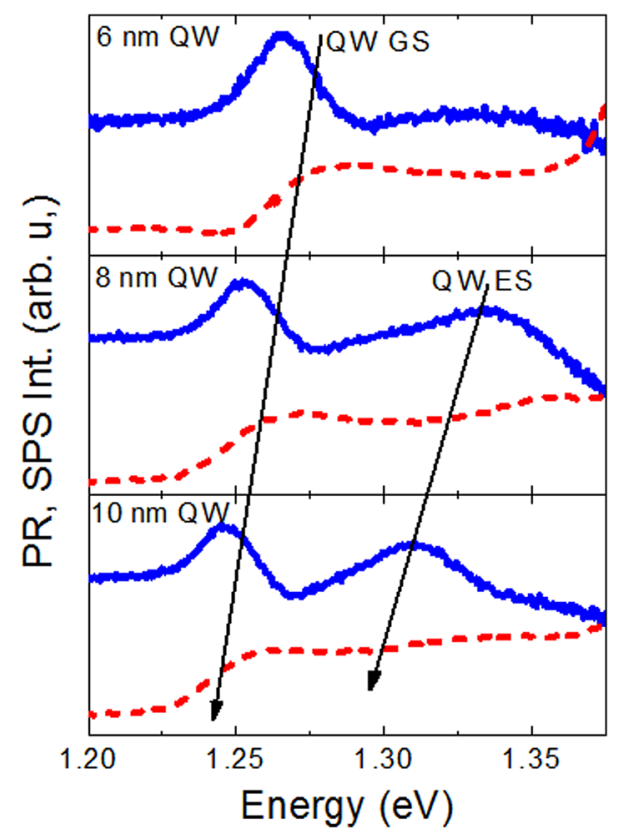

FIG. 1. Room temperature photoreflectance (solid blue lines) and surface photovoltage spectroscopy spectra (red dashed lines) for three $\mathrm{GaAs}_{0.988} \mathrm{~N}_{0.012} / \mathrm{GaAs} \mathrm{QWs}$ of different widths. The spectral features are assigned as QW GS and QW ES for the ground and excited state optical transitions, respectively. 
$1.2 \%$ and different QW widths of 6, 8, and $10 \mathrm{~nm}$. Using various widths for a constant composition is an approach which allows probing the appearance/disappearance of higher order state transitions in the spectra (due to the changed confinement width, the number of confined levels is altered), which eventually, after comparing to the results of calculations, allows determining the band gap discontinuities (only the proper discontinuity will give the agreement for the transition energies, and especially the number of confined state transitions for various QW widths). As can be seen in Fig. 1, there is only one spectral feature present for the narrowest QW (6 nm width), in both types of spectra, PR and SPS. This, even without the calculations, can be attributed to the fundamental transition in the well. This transition is observed for the wider QWs and shifts to the red with increasing the width, as expected. For the $8 \mathrm{~nm}$ wide QW, a second feature appears at about $80 \mathrm{meV}$ higher energy. And finally, this second transition is also observed for the case of the $10 \mathrm{~nm}$ wide QW, being red shifted and separation of which decreased to about $60 \mathrm{meV}$ with respect to the ground state transition, i.e. as expected with increasing the well layer thickness. It is worth noting that no other optical features could be observed, except of the GaAs-related bulk-like transition, which has been left out of the range of Fig. 1 for clarity (as this is much more intensive, and out of the interest for this discussion). In order to derive more exact spectral positions of the observed features, a respective analysis must be employed. In the case of the PR spectra (whose line shape is derivative-like), a fitting procedure according to the following formula has been performed:

$$
\frac{\Delta R}{R}(E)=\operatorname{Re}\left[C \cdot e^{i \cdot \vartheta}\left(E-E_{0}+i \cdot \Gamma\right)^{-m}\right],
$$

where $C$ and $\vartheta$ are the amplitude and phase of the spectral line, and $E_{0}$ and $\Gamma$ are the energy and the broadening parameter of the transition, respectively. The parameter $m$ refers to the type of an optical transition and critical point. We used here $\mathrm{m}=3$ as it has been proven to produce a line shape imitating well the first derivative of Gaussian function (appropriate for inhomogeneously broadened confined state transitions) and giving correct transition energies. An example of such fit is shown in Fig. 2(a) for the case of the $10 \mathrm{~nm}$ wide QW from Fig. 1. As the detailed line shape is also dependent on the phase parameter, the obtained energies, which are 1.253 and 1.328 , respectively, fall spectrally between the two maxima of the respective PR lines.

Similarly, the extraction of the transition energy directly from the SPS spectra is not very exact, especially for the higher energy transitions, which appear on the background of the absorption related to the fundamental one, and are not very pronounced (however visible) in the spectrum as measured (see Fig. 1). Therefore, the processing of the spectra is needed, and a respective fitting procedure afterwards. It has been $\operatorname{shown}^{23,32}$ that the numerically calculated derivative of $E \times S P S(E)$ with respect to the photon energy $E$, $d(E \times S P S(E) / d E$ (denoted as DSPS, i.e., a derivative of the SPS response) is proportional to $d(\alpha(E)) / d E$, where $\alpha$ is the absorption coefficient and resembles the line shape described by Eq. (1) but for parameter $m=2$. Such an analysis has

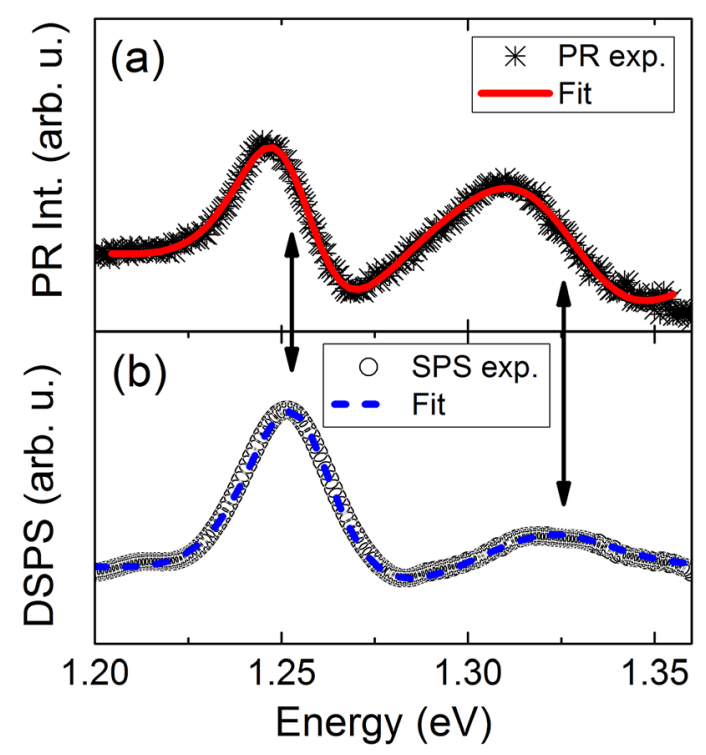

FIG. 2. Results of fitting procedure for the example of $10 \mathrm{~nm}$ well from Fig. 1, for both PR and DSPS spectra.

been used and its result is shown in Fig. 2(b). As the phase parameter is by the definition different in the case of these spectra and the PR ones, the obtained energies, which are very close to the ones from PR $(1.251$ and $1.323 \mathrm{eV})$, fall more onto the respective peaks of the derivative curve of the DSPS signal. The second higher energy transition is also a bit less pronounced in DSPS, but generally, both the types of the spectra confirm the existence and the energy positions of the observed optical transitions.

Analogous spectra have also been recorded and their analysis performed for the second series of structures with $2.2 \%$ of nitrogen.

\section{B. Band offset}

The experimental data of the previous chapter is compared with the results of calculations within the theoretical model presented in Sec. III. We calculated the transition energies in $\mathrm{GaAs}_{1-\mathrm{x}} \mathrm{N}_{\mathrm{x}} / \mathrm{GaAs}$ QWs with $\mathrm{N}$ contents of $\mathrm{x}=1.2 \%$ and $2.2 \%$, and versus the well width in the range of at least $6-10 \mathrm{~nm}$. The chemical band offsets (band gap discontinuities), i.e., the ones between the unstrained materials (or before the application of the strain), are the input parameters, and treated as semi-free, i.e., tunable in order to match the theoretical dependencies with the experimental data. Figure 3 compares the experimentally determined transition energies of a series of $\mathrm{GaAs}_{1-\mathrm{x}} \mathrm{N}_{\mathrm{x}} / \mathrm{GaAs}$ QWs with $\mathrm{x}=1.2 \%$ and various well widths with those calculated using the 10-band $\mathbf{k} \cdot \mathbf{p}$ Hamiltonian. The electron effective mass was taken from the BAC model ${ }^{18}$ in the first approximation. The conduction band offsets used in the calculations were equal to $70 \%$ (red line), $80 \%$ (blue line), and 90\% (green line), respectively. Using that limited range is based on what has been reported in the literature previously ${ }^{16,21}$ and also on our previous work, which predicted that the properties of more complex structures of InGaAsN/GaAsN/GaAs QWs will strongly depend on that if the GaAsN/GaAs band offset is 


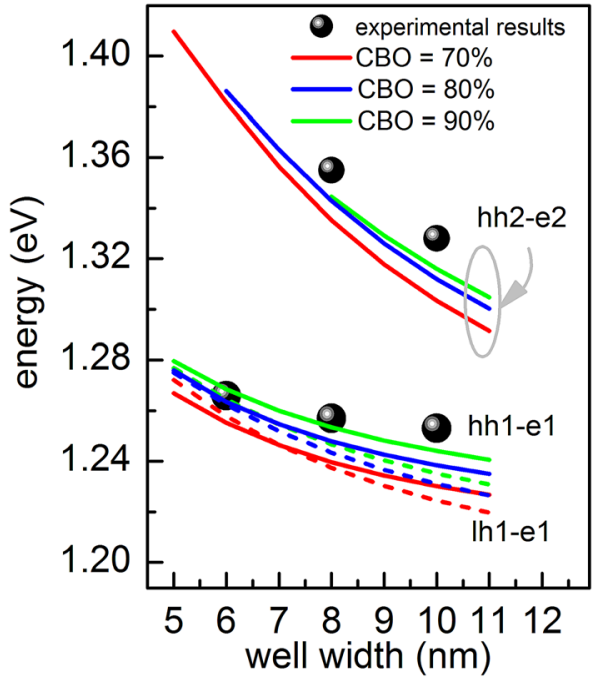

FIG. 3. Well width dependence of the transition energies of $\mathrm{GaAs}_{0.988} \mathrm{~N}_{0.012} / \mathrm{GaAs}$ quantum wells calculated by using the 10-band $\mathbf{k} \cdot \mathbf{p}$ Hamiltonian if the electron effective mass is from BAC model (Ref. 18). The red, blue, and green lines (solid-heavy hole transitions; dashed-light hole transitions) represent the calculated values if the conduction band offset are equal to $70 \%, 80 \%$, and $90 \%$, respectively.

closer to $70 \%$ or $90 \%$. As we see, indeed an unambiguous determination of the band offset is hardly possible when following just the ground state transition, which is weakly sensitive to that parameter of the structure. Additionally, which should be expected in the case of these tensile strained QWs, there coexist two closely spaced transitions in the range of the lowest energy spectral feature: a light hole related transition (i.e. between the fundamental light hole confined level and fundamental electron confined level-lh1-e1) and at slightly higher energy a heavy hole transition (the fundamental heavy hole confined level and the fundamental electron confined level-hh1-e1). While considering only the transitions at $\mathbf{k}=0$, we can still use the names as in the single band model, i.e., "heavy hole" or "light hole" bands. The superposition of the two transitions at the ground state energy makes the interpretation even more difficult, especially as these two transitions are separated by only a few $\mathrm{meV}$ and cannot be resolved in the broadened spectra (the line broadening is about $20 \mathrm{meV}$ ) of rather strongly inhomogeneous system of dilute nitride. However, one can assume that the experimental spectra are mostly dominated by the heavy hole transitions having larger oscillator strength (by approximately a factor of 3 in the quantum well).

The higher order transitions change much stronger when the band offsets are altered and the "lh" and "hh" ones are better separated. When analyzing the dependencies plotted in Fig. 3, we observe that the calculated hh2-e2 transition (second heavy hole level and second electron level) occurs very close to the energies of the experimental higher energy spectral feature (called QW ES in Fig. 1). Although the absolute energies do not agree very exactly, one can still follow the tendency and number of transitions expected in the spectra. The latter brings us to the conclusion that the conduction band offset must be taken from the range of $80 \%-90 \%$ for $\mathrm{N}$ content of $1.2 \%$, because for $80 \%$ or smaller values a second optical transition should also be present in the spectra for the $6 \mathrm{~nm}$ wide QW, which is not the case. On the other hand, the second transition could not be seen for the $8 \mathrm{~nm}$ wide QW, if the conduction band offset is $\geq 90 \%$. This result remains in agreement with some of the previous considerations. ${ }^{16,21}$ Therefore, following the dependence proposed in Ref. 16, we obtain that the conduction band offsets could be about $86 \%$ for $\mathrm{x}=1.2 \%$. When analyzing similarly the data for the larger $\mathrm{N}$ content, and assuming the dependence from Ref. 16 is still valid, we obtain the conduction band offset ratio of about $83 \%$ for $\mathrm{x}=2.2 \%$. These two offset values are then used in further calculations.

\section{Electron effective mass}

In order to improve the agreement between the theoretical and experimental results, we tune the electron effective mass, which is the second parameter which is not very well defined in the literature. Figure 4 shows the calculated transition energies in $\mathrm{GaAs}_{1-\mathrm{x}} \mathrm{N}_{\mathrm{x}} / \mathrm{GaAs}$ QWs for two different $\mathrm{N}$ mole fractions $\mathrm{x}=1.2 \%$ (a) and $\mathrm{x}=2.2 \%$ (b) as a function of the QW width. The red dashed lines show results obtained using the theoretical model presented in Ref. 33, while the black full dots correspond to our experimental results. In order to improve the agreement with respect to the experimental data, the transition energies have also been calculated for the corrected electron effective massin Fig. 4. For the two considered cases of $\mathrm{N}$ content, the effective electron mass has been set to be equal to $0.09 \mathrm{~m}_{\mathrm{o}}$ for the sample with $1.2 \% \mathrm{~N}$ and $0.15 \mathrm{~m}_{\mathrm{o}}$ for the sample with $2.2 \% \mathrm{~N}$. In the second case, this is more than twice larger than the electron effective mass in GaAs. Suggestions on the necessity to increase the effective mass in GaAsN have already been made in several papers previously. ${ }^{13,16,19-21,37}$ Hai and co-workers ${ }^{19}$ determined the mass values to be $0.12 \mathrm{~m}_{\mathrm{o}}$ and $0.19 \mathrm{~m}_{\mathrm{o}}$ for $7 \mathrm{~nm} \mathrm{GaAs} \mathrm{N}_{\mathrm{x}} \mathrm{N}_{1-\mathrm{x}} / \mathrm{GaAs}$ QWs with $\mathrm{x}=1.2 \%$ and $2 \%$, respectively. Authors in Ref. 20 studied samples with a similar nitrogen content of $1.2 \%<\mathrm{x}<2.8 \%$ and different $\mathrm{GaAs}_{\mathrm{x}} \mathrm{N}_{1-\mathrm{x}}$ well widths from 3 to $9 \mathrm{~nm}$ and showed an increase in the electron effective mass to about $0.11 \mathrm{~m}_{\mathrm{o}}$. Also in Ref. 21, it has been indicated that for
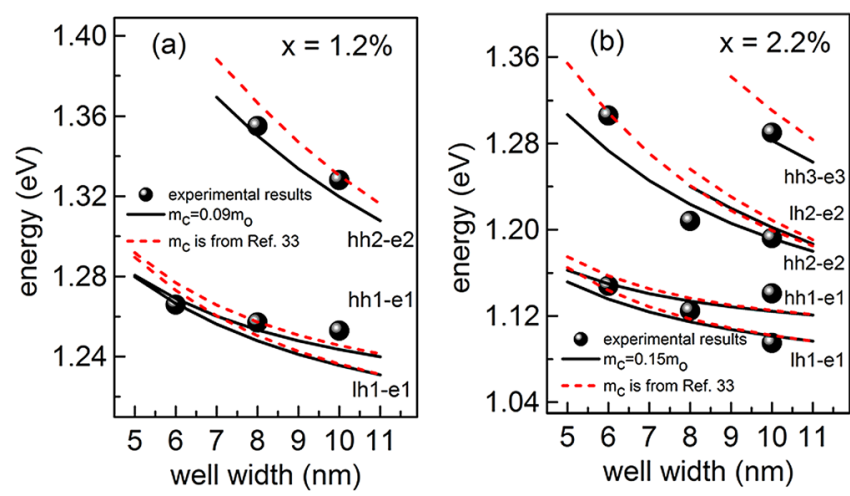

FIG. 4. Well width dependence of the transition energies of $\mathrm{GaAs}_{0.988} \mathrm{~N}_{0.012} / \mathrm{GaAs}$ (a) and $\mathrm{GaAs}_{0.978} \mathrm{~N}_{0.022} / \mathrm{GaAs}$ (b) quantum well calculated by using the 10-band $\mathbf{k} \cdot \mathbf{p}$ Hamiltonian if the electron effective mass is from Ref. 33 (dashed red line) and if the electron effective mass is equal to 0.09 for $\mathrm{x}=1.2 \% \mathrm{~N}$ (a) and 0.15 for $\mathrm{x}=2.2 \% \mathrm{~N}$ (b) (solid black line). 


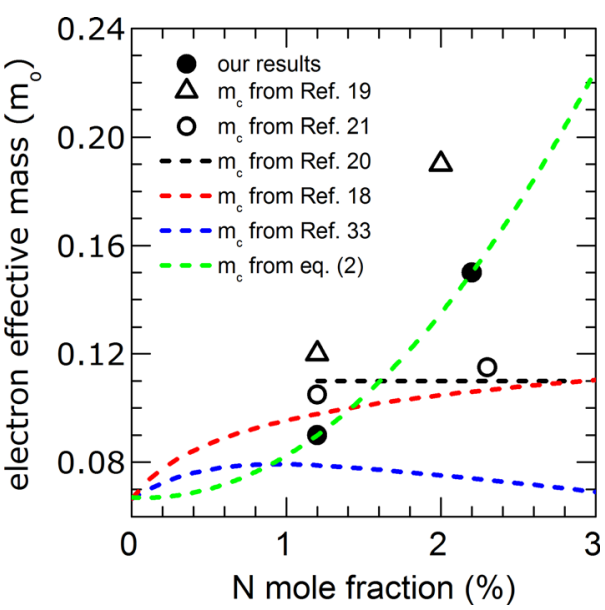

FIG. 5. Effect of increasing $\mathrm{N}$ mole fraction on the electron effective mass. Data points: experimental mass values taken from Ref. $19(\Delta)$, Ref. $21(\bigcirc)$, our results $(\mathbf{O})$. The dashed black lines presents results from Ref. 20. The dashed red line presents calculated values from BAC model (Ref. 18). The dashed blue line presents results from Ref. 33. The dashed green line, calculated values with $\mathrm{GaAs}_{1-\mathrm{x}} \mathrm{N}_{\mathrm{x}} / \mathrm{GaAs} \mathrm{QW}$ electron effective mass given by Eq. (2).

$\mathrm{x}=1.2 \%$ the electron effective mass is $0.105 \mathrm{~m}_{\mathrm{o}}$ and for $\mathrm{x}=2.3 \%$ it has a value of $0.115 \mathrm{~m}_{\mathrm{o}}$. Finally, our results of electron effective mass are a bit lower than those reported in Ref. 13. The previously existing descriptions for the $\mathrm{N}$ mole fraction from $0 \%$ to $3 \%$ give the results presented in Fig. 5. The red dashed line concerns the results calculated using the BAC model, ${ }^{18}$ while the black dashed line corresponds to the results obtained based on the model proposed in Ref. 16 . The calculated values of effective electron mass are increased compared to GaAs, but still lower than those directly measured or determined indirectly using the optical experiments. This discrepancy is especially significant for the $\mathrm{N}$ mole fraction larger than $2 \%$. This is described in Ref. 37, in which it has been found by the ab initio LMTO (Linear Muffin Tin Orbital) calculations that the values obtained for the electron effective mass at the bottom of the conduction band should be in the range from $0.065 \mathrm{~m}_{\mathrm{o}}$ to $0.12 \mathrm{~m}_{\mathrm{o}}$ for $0.009<\mathrm{x}<0.04$. In our case, and based on the comparison to the experimental results, we approximated the electron effective mass in a function of the $\mathrm{N}$ content by the following expression:

$$
\frac{m_{e}^{*}(x)}{m_{o}}=b_{o}+b_{1} x+b_{2} x^{2},
$$

where $b_{o}=0.067, b_{1}=-0.31$, and $\mathrm{b}_{2}=185.6$. However, some further work and additional and independent experiments are still needed to confirm such a dependence for more values of the $\mathrm{N}$ contents, also out of the range of compositions considered here.

\section{SUMMARY}

In summary, detecting the optical transitions in the $\mathrm{GaAs}_{1-x} \mathrm{~N}_{\mathrm{x}} / \mathrm{GaAs}$ quantum wells by photoreflectance and surface photovoltage spectroscopy has allowed studying the details of the band structure of this dilute nitride material system. The dependencies of the transition energies on the well width and $\mathrm{N}$ concentration have been investigated. Based on the comparison of the experimental data with the results of the calculations from the 10-band $\mathbf{k} \cdot \mathbf{p}$ modeling, we could estimate the conduction band offset to be equal $86 \%( \pm 4 \%)$ for $\mathrm{x}=1.2 \%$ and $83 \%( \pm 3 \%)$ for $\mathrm{x}=2.2 \%$. The results suggest additionally an increase in the electron effective mass to about $0.09 m_{\mathrm{o}}$ for $\mathrm{x}=0.012$ and $0.15 m_{\mathrm{o}}$ for $\mathrm{x}=0.022$. Both the parameters need to be considered accordingly in order to predict properly on the electronic levels of more complex GaAs-based structures containing the GaAsN alloy. Eventually, the obtained band offset confirmed the expectations on the enhancement of the oscillator strength of the optical transitions in InGaAsN/GaAsN/GaAs $\mathrm{QWs}^{9}$ designed to be employed for the formation of exciton polaritons in the telecommunication range. This makes the material system indeed promising for practical exploitation of the quantum-electrodynamics-based optoelectronic applications.

\section{ACKNOWLEDGMENTS}

The authors acknowledge the financial support from the bilateral project of Deutsche Forschungsgemeinschaft (Project named LIEPOLATE) and Polish Ministry of Science and Higher Education (Project No. DPN/N99/DFG/2010), and the COPERNICUS Award of the Foundation for Polish Science and Deutsche Forschungsgemeinschaft.

\section{APPENDIX: TEN-BAND k.p MODEL}

In the 10-band scheme, the total wave function of the subband $n$ in the QW can be written as

$$
\Psi_{n}(r)=\sum_{j=1}^{10} F_{n, j}(r) u_{j}(r)
$$

where $F_{n, j}(r)$ is the envelope function and $u_{j}(r)$ is the periodic part of the Bloch basis function at the zone center. The following Bloch basis functions for the high-lying N-band ( $\mathrm{N}$ will be used to assign the nitrogen related states), the conduction band $(\mathrm{CB})$, and the heavy hole $(\mathrm{HH})$, the light hole ( $\mathrm{LH})$, and the spin-orbit split-off (SO) bands have been used to derive the 10-band $\mathbf{k} \cdot \mathbf{p}$ Hamiltonian

$$
\begin{gathered}
\left|u_{1}\right\rangle=\left|\frac{1}{2},+\frac{1}{2}\right\rangle=\left|s_{N} ; \uparrow\right\rangle, \\
\left|u_{2}\right\rangle=\left|\frac{1}{2},+\frac{1}{2}\right\rangle=|s ; \uparrow\rangle,
\end{gathered}
$$

$$
\left|u_{3}\right\rangle=\left|\frac{3}{2},+\frac{3}{2}\right\rangle=\frac{i}{\sqrt{2}}[|x ; \uparrow\rangle+i|y ; \uparrow\rangle],
$$

$$
\begin{gathered}
\left|u_{4}\right\rangle=\left|\frac{3}{2},+\frac{1}{2}\right\rangle=\frac{i}{\sqrt{6}}[|x ; \downarrow\rangle+i|y ; \downarrow\rangle-2|z ; \uparrow\rangle], \\
\left|u_{5}\right\rangle=\left|\frac{1}{2},+\frac{1}{2}\right\rangle=\frac{i}{\sqrt{3}}[|x ; \downarrow\rangle+i|y ; \downarrow\rangle+|z ; \uparrow\rangle],
\end{gathered}
$$




$$
\begin{gathered}
\left|u_{6}\right\rangle=\left|\frac{1}{2},-\frac{1}{2}\right\rangle=-\left|s_{N} ; \downarrow\right\rangle, \\
\left|u_{7}\right\rangle=\left|\frac{1}{2},+\frac{1}{2}\right\rangle=-|s ; \downarrow\rangle, \\
\left|u_{8}\right\rangle=\left|\frac{3}{2},-\frac{3}{2}\right\rangle=-\frac{i}{\sqrt{2}}[|x ; \downarrow\rangle-i|y ; \downarrow\rangle], \\
\left|u_{9}\right\rangle=\left|\frac{3}{2},-\frac{1}{2}\right\rangle=+\frac{i}{\sqrt{6}}[|x ; \uparrow\rangle-i|y ; \uparrow\rangle+2|z ; \downarrow\rangle],
\end{gathered}
$$

$$
\left|u_{10}\right\rangle=\left|\frac{1}{2},-\frac{1}{2}\right\rangle=+\frac{i}{\sqrt{3}}[|x ; \uparrow\rangle-i|y ; \uparrow\rangle-|z ; \downarrow\rangle]
$$

where $\left|S_{N}\right\rangle$ denotes the s-like $\mathrm{N}$ Bloch state, $|S\rangle$ denotes the s-like CB Bloch state, and $|x\rangle,|y\rangle,|z\rangle$ denotes the p-like VB Bloch states. The 10-band k·p Hamiltonian, which includes the coupling of $\mathrm{N}, \mathrm{CB}, \mathrm{HH}, \mathrm{LH}$, and $\mathrm{SO}$ into account is given below

$$
\mathrm{H}=\left[\begin{array}{llllllllll}
E_{N} & V_{N C} & 0 & 0 & 0 & 0 & 0 & 0 & 0 & 0 \\
& E_{C B} & -\sqrt{3} T_{+} & \sqrt{2} U & -U & 0 & 0 & 0 & -T_{-} & -\sqrt{2} T_{-} \\
& & E_{H H} & \sqrt{2} S & -S & 0 & 0 & 0 & -R & -\sqrt{2} R \\
& & & E_{L H} & Q & 0 & T_{+}^{*} & R & 0 & \sqrt{3} S \\
& & & & E_{S O} & 0 & \sqrt{2} T_{+}^{*} & \sqrt{2} R & -\sqrt{3} S & 0 \\
& & & & & E_{N} & V_{N C} & 0 & 0 & 0 \\
& & & & & & E_{C B} & -\sqrt{3} T_{-} & \sqrt{2} U & -U \\
& & & & & & & E_{H H} & \sqrt{2} S^{*} & -S^{*} \\
& & & & & & & & E_{L H} & Q \\
& & & & & & & & & E_{S O}
\end{array}\right],
$$

where

$$
\begin{gathered}
E_{N}=E_{N o}-\gamma x+\kappa x+\delta E_{N}^{h y d}, \\
E_{C B}=E_{C o}-\alpha x+\kappa x+\frac{\hbar^{2}}{2 m_{o}} s\left(k_{x}^{2}+k_{y}^{2}+k_{z}^{2}\right)+\delta E_{C B}^{h y d}, \\
E_{H H}=E_{v o}+\kappa x-\frac{\hbar^{2}}{2 m_{o}}\left(\gamma_{1}+\gamma_{2}\right)\left(k_{x}^{2}+k_{y}^{2}\right) \\
-\frac{\hbar^{2}}{2 m_{o}}\left(\gamma_{1}-2 \gamma_{2}\right) k_{z}^{2}+\delta E_{V B}^{h y d}-\eta_{a x}, \\
E_{L H}=E_{V o}+\kappa x-\frac{\hbar^{2}}{2 m_{o}}\left(\gamma_{1}-\gamma_{2}\right)\left(k_{x}^{2}+k_{y}^{2}\right) \\
-\frac{\hbar^{2}}{2 m_{o}}\left(\gamma_{1}+2 \gamma_{2}\right) k_{z}^{2}+\delta E_{V B}^{h y d}+\eta_{a x}, \\
E_{S O}=E_{V o}+\kappa x-\Delta_{S O}-\frac{\hbar^{2}}{2 m_{o}} \gamma_{1}\left(k_{x}^{2}+k_{y}^{2}\right)-\frac{\hbar^{2}}{2 m_{o}} \gamma_{1} k_{z}^{2} \\
+\delta E_{V B}^{h y d}, \quad \\
T_{ \pm}=\frac{1}{\sqrt{6}} P\left(k_{x} \pm i k_{y}\right), \\
U=\frac{1}{\sqrt{3}} P k_{z}, \\
\sqrt{\frac{3}{2} \frac{\hbar^{2}}{m_{o}} \gamma_{3} k_{z}\left(k_{x}-i k_{y}\right),}
\end{gathered}
$$

$$
\begin{gathered}
R=\frac{\sqrt{3}}{2} \frac{\hbar^{2}}{2 m_{o}}\left[\left(\gamma_{2}+\gamma_{3}\right)\left(k_{x}-i k_{y}\right)^{2}-\left(\gamma_{3}-\gamma_{2}\right)\left(k_{x}+i k_{y}\right)^{2}\right] \\
Q=-\frac{1}{\sqrt{2}} \frac{\hbar^{2}}{m_{o}} \gamma_{2} k_{\|}^{2}+\sqrt{2} \frac{\hbar^{2}}{m_{o}} \gamma_{2} k_{z}^{2}-\sqrt{2} \eta_{a x}, \\
k_{\|}^{2}=k_{x}^{2}+k_{y}^{2},
\end{gathered}
$$

and $V_{N}=\beta \sqrt{x}$ describes the coupling between the $\mathrm{N}$ states and conduction band edge, $\beta$ is the interaction factor between $\mathrm{N}$ and $\mathrm{CB}, \mathrm{E}_{\mathrm{No}}, \mathrm{E}_{\mathrm{Co}}, \mathrm{E}_{\mathrm{Vo}}$ are the energies of $\mathrm{N}, \mathrm{CB}$, $\mathrm{VB}$, respectively, $\Delta_{\mathrm{SO}}$ is the spin-orbit splitting energy, $\mathrm{k}_{\mathrm{x}}$ and $\mathrm{k}_{\mathrm{y}}$ are the wave vectors in the plane, $\mathrm{k}_{\mathrm{z}}$ is the wave vector along the QW growth direction. The term $s=1 / m_{c}^{*}$ $-\left(E_{P} / 3\right)\left[\frac{2}{E_{g}}+1 /\left(E_{g}+\Delta_{S O}\right)\right]$ is used in place of $1 / m_{c}^{*} \cdot \gamma_{1}$, $\gamma_{2}$, and $\gamma_{3}$ are the modified Luttinger parameters $\gamma_{1}=\gamma_{1}^{L}-E_{P} /\left(3 E_{g}^{h}\right), \gamma_{2,3}=\gamma_{2,3}^{L}-E_{P} /\left(6 E_{g}^{h}\right) . E_{P}$ and $E_{g}^{h}$ are the optical matrix parameter and band gap of the host material, respectively. $P$ is the Kane matrix element for the conduction band $\left(E_{P}=2 m_{o} P^{2} / \hbar^{2}\right) . \quad \delta E_{C B}^{h y d}=-2 a_{i}\left(1-\frac{c_{12}}{c 1}\right) \varepsilon_{x x}$ and $\eta_{a x}=-b_{a x}\left(1+\frac{2 c_{12}}{c_{11}}\right) \varepsilon_{x x}$ describe the influence of the hydrostatic and shear strain components on the band structure, where $a_{i}$ are the hydrostatic deformation potentials (with index $i=\mathrm{N}, \mathrm{CB}, \mathrm{VB}), c_{11}$ and $c_{12}$ are the elastic constants, $b_{a x}$ is the axial deformation potential and $\varepsilon_{x x}$ is the strain in the layer. The parameters $\alpha$ and $\beta$ describe the band gap reduction due to the introduction of $\mathrm{N}$, while $\kappa$ takes into account the variation of the VB offset between unstrained $\mathrm{GaN}_{\mathrm{x}} \mathrm{As}_{1-\mathrm{x}}$ and GaAs. And finally, $x$ is the fraction of $\mathrm{N}$ in the structure. The band parameters of the parental binary compounds used in our calculations are taken after Ref. 16. 
We quantize the Hamiltonian along the growth direction (z-axis) and we replace the $\mathrm{z}$ component of $\mathbf{k}$ vector by the operator $k_{z} \rightarrow-i \partial / \partial z$. Then, we denote the in-plane wave vector by $\boldsymbol{k}_{\|}=\left[k_{x}, k_{y}\right]$. We solve the 10 -band $\mathbf{k} \cdot \mathbf{p}$ Hamiltonian numerically using the finite difference technique. ${ }^{35}$

${ }^{1}$ M. Weyers, M. Sato, and H. Ando, Jpn. J. Appl. Phys. 31, L853-L855 (1992).

${ }^{2}$ S.-H. Wei and A. Zunger, Phys. Rev. Lett. 76, 664 (1996).

${ }^{3}$ J. W. Ager III and W. Walukiewicz, Semicond. Sci. Technol. 17, 741 (2002).

${ }^{4}$ R. Oshima, A. Takata, and Y. Okada, Appl. Phys. Lett. 93, 083111 (2008).

${ }^{5}$ D. Gollub, S. Moses, and A. Forchel, IEEE J. Quant. Elect. 40(4), 337-342 (2004).

${ }^{6} \mathrm{M}$. Le Dû, J.-C. Harmand, O. Mauguin, L. Largeau, L. Travers, and J.-L. Oudar, Appl. Phys. Lett. 88, 201110 (2006).

${ }^{7}$ N. Tansu, J.-Y. Yeh, and Luke J. Mawst, Appl. Phys. Lett. 83, 2512 (2003).

${ }^{8}$ D. Bisping, D. Pucicki, M. Fischer, S. Höfling, and A. Forchel, J. Cryst. Growth 311, 1715 (2009).

${ }^{9}$ K. Ryczko, G. Sęk, J. Misiewicz, F. Langer, S. Höfling, and M. Kamp, J. Appl. Phys. 111, 123503 (2012).

${ }^{10}$ S. Sakai, Y. Ueta, and Y. Terauchi, Jpn J. Appl. Phys., Part 1 32, 4413 (1993).

${ }^{11}$ T. Kitani, M. Kondow, T. Kikawa, Y. Yazawa, M. Okai, and K. Uomi, Jpn J. Appl. Phys., Part 1 38, 5003 (1999).

${ }^{12}$ L. Bellaiche, S.-H. Wei, and A. Zunger, Phys. Rev. B 56, 10233 (1997).

${ }^{13}$ Y. Zhang, A. Mascarenhas, H. P. Xin, and C. W. Tu, Phys. Rev. B 61, 7479 (2000).

${ }^{14}$ I. A. Buyanova, G. Pozina, P. N. Hai, W. M. Chen, H. P. Xin, and C. W. Tu, Phys. Rev. B 63, 033303 (2000).

${ }^{15}$ P. Krispin, S. G. Spruytte, J. S. Harris, and K. H. Ploog, J. Appl. Phys. 90, 2405 (2001).

${ }^{16}$ S. Tomić, E. P. O'Reilly, P. J. Klar, H. Gruning, W. Heimbrodt, W. M. Chen, and I. A. Buyanova, Phys. Rev. B 69, 245305 (2004).

${ }^{17}$ J.-R. Lee, Y.-Y. Chen, C.-R. Lu, W.-I. Lee, and S.-C. Lee, Mater. Sci. Eng. B 100, 248 (2003)
${ }^{18}$ W. Walukiewicz, W. Shan, J. W. Ager III, D. R. Chamberlin, E. E. Haller, J. F. Geisz, D. J. Friedman, J. M. Olson, and S. R. Kurtz, in Proceedings of the 195th Electrochemical Society Meeting, Seattle, WA (The Electrochemical Society, Pennington, 1999), Vol. 99-11, p. 190.

${ }^{19}$ P. N. Hai, W. M. Chen, I. A. Buyanova, H. P. Xin, and C. W. Tu, Appl, Phys. Lett. 77, 1843 (2000).

${ }^{20}$ J. Wu, W. Shan, W. Walukiewicz, K. M. Yu, J. W. Ager III, E. E. Haller, H. P. Xin, and C. W. Tu, Phys. Rev. B 64, 085320 (2001).

${ }^{21}$ R. Kudrawiec, M. Motyka, M. Gładysiewicz, J. Misiewicz, J. A. Gupta, and G. C. Aers, Solid State Commun. 138, 365 (2006).

${ }^{22}$ T. Dannecker, Y. Jin, H. Cheng, C. F. Gorman, J. Buckeridge, C. Uher, S. Fahy, C. Kurdak, and R. S. Goldman, Phys. Rev. B 82, 125203 (2010).

${ }^{23}$ S. Datta, B. M. Arora, and S. Kumar, Phys. Rev. B 62, 13604 (2000).

${ }^{24}$ P. Sitarek, H. P. Hsu, Y. S. Huang, J. M. Lin, H. H. Lin, and K. K. Tiong, J. Appl. Phys. 105, 123523 (2009)

${ }^{25}$ G. Sęk, M. Motyka, K. Ryczko, F. Janiak, J. Misiewicz, S. Belahsene, G. Boissier, and Y. Rouillard, Jpn. J. Appl. Phys., Part 1 49, 031202 (2010).

${ }^{26}$ R. Kudrawiec and J. Misiewicz, J. Phys.: Conf. Ser. 146, 012029 (2009).

${ }^{27}$ M. Motyka, R. Kudrawiec, J. Misiewicz, M. Hümmer, K. Rößner, T. Lehnhardt, M. Müller, and A. Forchel, J. Appl. Phys. 103, 113514 (2008).

${ }^{28}$ M. Motyka, G. Sęk, R. Kudrawiec, J. Misiewicz, L. H. Li, and A. Fiore, J. Appl. Phys. 100, 073502 (2006).

${ }^{29}$ R. Kudrawiec, G. Sęk, K. Ryczko, J. Misiewicz, and J. C. Harmand, Appl. Phys. Lett. 84, 3453 (2004).

${ }^{30}$ G. Sęk, K. Ryczko, M. Kubisa, J. Misiewicz, M. Bayer, T. Wang, J. Koeth, and A. Forchel, Thin Solid Films 364, 220 (2000).

${ }^{31}$ R. Kudrawiec and J. Misiewicz, Rev. Sci. Instrum. 80, 096103 (2009).

${ }^{32}$ S. Datta, S. Ghosh, and B. M. Arora, Rev. Sci. Instrum. 72, 177 (2001).

${ }^{33}$ S. Tomić, E. P. O'Reilly, R. Fehse, S. J. Sweeney, A. R. Adams, A. D. Andreev, S. A. Choulis, T. J. C. Hosea, and H. Riechert, IEEE J. Sel. Top. Quantum Electron. 9, 1228 (2003).

${ }^{34}$ S. T. Ng, W. J. Fan, Y. X. Dang, and S. F. Yoon, Phys. Rev. B 72, 115341 (2005).

${ }^{35}$ J. W. Thomas, Numerical Partial Differential Equations (Springer, New York, 1995).

${ }^{36}$ N. Bachrach-Ashkenasy, L. Kronik, Y. Shapiraa, Y. Rosenwaks, M. C. Hanna, M. Leibovitch, and P. Ram, Appl. Phys. Lett. 68, 879 (1996).

${ }^{37}$ C. Skierbiszewski, I. Gorczyca, S. P. Łepkowski, J. Łusakowski, J. Borysiuk, and J. Toivonen, Semicond. Sci. Technol. 19, 1189 (2004). 